\title{
Video Article \\ Isolation of Single Intracellular Bacterial Communities Generated from a Murine Model of Urinary Tract Infection for Downstream Single-cell Analysis
}

\author{
EnJun Yang ${ }^{1}$, Jacqueline L.Y. Chee ${ }^{1}$, Suhanya Duraiswamy ${ }^{2}$, Siyi Chen ${ }^{3}$, Kristin Lees ${ }^{3}$, Swaine L. Chen ${ }^{1,3}$ \\ ${ }^{1}$ Infectious Diseases Group, Genome Institute of Singapore \\ ${ }^{2}$ Department of Chemical and Biomolecular Engineering, National University of Singapore \\ ${ }^{3}$ Division of Infectious Diseases, Department of Medicine, Yong Loo Lin School of Medicine, National University of Singapore
}

Correspondence to: Swaine L. Chen at slchen@gis.a-star.edu.sg

URL: https://www.jove.com/video/58829

DOI: doi: $10.3791 / 58829$

Keywords: Immunology and Infection, Issue 146, urinary tract infection, single-cell isolation, intracellular bacterial communities, uropathogenic Escherichia coli, murine, UTI

Date Published: 4/16/2019

Citation: Yang, E., Chee, J.L., Duraiswamy, S., Chen, S., Lees, K., Chen, S.L. Isolation of Single Intracellular Bacterial Communities Generated from a Murine Model of Urinary Tract Infection for Downstream Single-cell Analysis. J. Vis. Exp. (146), e58829, doi:10.3791/58829 (2019).

\section{Abstract}

In this article, we outline a procedure used to isolate individual intracellular bacterial communities from a mouse that has been experimentally infected in the urinary tract. The protocol can be broadly divided into three sections: the infection, bladder epithelial cell harvesting, and mouth micropipetting to isolate individual infected epithelial cells. The isolated epithelial cell contains viable bacterial cells and is nearly free of contaminating extracellular bacteria, making it ideal for downstream single-cell analysis. The time taken from the start of infection to obtaining a single intracellular bacterial community is about $8 \mathrm{~h}$. This protocol is inexpensive to deploy and uses widely available materials, and we anticipate that it can also be utilized in other infection models to isolate single infected cells from cell mixtures even if those infected cells are rare. However, due to a potential risk in mouth micropipetting, this procedure is not recommended for highly infectious agents.

\section{Video Link}

The video component of this article can be found at https://www.jove.com/video/58829/

\section{Introduction}

Urinary tract infections (UTIs) are one of the most common bacterial infections. An estimated $40-50 \%$ of women are expected to experience at least one urinary tract infection (UTI) during their lifetime ${ }^{1}$. One of the main agents of UTI is uropathogenic E. coli (UPEC), which accounts for over $70 \%$ of uncomplicated UTIs ${ }^{2}$. Furthermore, approximately one quarter of those who have a UTI will have a recurrent infection, often caused by the same strain, despite appropriate antibiotic treatment ${ }^{3}$. The high incidence of UTI represents a substantial burden on healthcare systems, costing more than $\$ 2$ billion a year in the US ${ }^{4}$. Furthermore, the use of antibiotics to treat UTIs also leads to rising antibiotic resistance rates, which is a major public health concern ${ }^{5}$.

Therefore, a large effort has been placed into understanding the mechanisms by which UPEC infects the urinary tract, as well as its ability to cause recurrent infections $s^{6,7,8}$. In particular, a mouse model of infection has been used to examine bacterial and host characteristics that contribute to $\mathrm{UTI}^{8}$. This mouse model has the benefit of being applicable to unmodified clinical strains isolated from human patients. This model has also led to the discovery of potentially druggable bacterial pathways important for establishment of UTI, such as the Type 1 pilus ${ }^{9}$ and iron acquisition systems ${ }^{10}$.

Compared to these successes in studying the early events in UTI, knowledge of the mechanisms underlying recurrent UTI is still lacking ${ }^{11}$. One hypothesis is that UPEC evades antibiotic therapy and causes recurrent infections in the bladder by forming intracellular bacterial communities (IBCs) within bladder epithelial cells. IBCs have been identified both in murine models of infection and in human UTI patients ${ }^{12,13}$. The presence of IBCs in urine samples from pediatric UTI patients has been associated with higher rates of recurrence ${ }^{14,15}$. However, isolating IBCs and studying the bacteria within them has proven to be technically challenging due to their rarity; it is estimated that an infected murine bladder typically only has $10-100 \mathrm{IBCs}^{16}$. Furthermore, bladder epithelial cells are relatively large $(50-120 \mu \mathrm{m})^{17}$, making it challenging to deploy fluorescence assisted cell sorting (FACS) given that typical FACS nozzles are designed with diameters of $70 \mu \mathrm{m}$ or $100 \mu \mathrm{m}$. Thus, cells as large as bladder epithelial cells are often removed by filtration prior to FACS to avoid clogging the fluidics.

Our lab recently described a general and economical method to isolate rare infected cells from mixtures such as scraped epithelial cells of the bladder ${ }^{18}$. To effectively isolate IBCs, we used traditional mouth pipetting. Mouth micropipetting is a technique that has long been used for micromanipulation of single cells and embryos for downstream analysis ${ }^{19,20,21,22,23,24,25}$. Traditional mouth pipetting of large liquid volumes (in milliliters) has often been the cause of laboratory related accidents, and the technique has rightly been shunned by much of the research community outside of traditional embryology and single cell applications. Our protocol is inspired by the single cell versions of this technique ${ }^{19,20}$ which mitigate risk by providing a large buffer $(>2 \mathrm{~mL})$ of air between the researcher and sample compared to the volume of liquid transferred $(<1 \mu \mathrm{L})$. This method also takes advantage of the fine control that mouth micropipetting provides, which translates to a low final volume of 
surrounding solution transferred and high purity of isolated cells. The technique uses inexpensive materials $(<\$ 50)$, and thus should be feasible to implement in all labs.

This visual protocol describes our IBC isolation technique, providing a reference to assist other researchers seeking to replicate this technique. The researcher will need access to a fluorescent dissecting microscope (or similar equipment) that can be used to visualize individual epithelial cells and the fluorescent bacteria during live imaging, with an open and accessible imaging stage for micropipetting (see the Table of Materials for the details of the microscope used, though other equivalent instrument models may also be used). While this protocol will focus on IBCs in a murine model of UTI, similar methods should be applicable to isolate infected cells from cell suspensions in other models of infection.

Protocol

All methods described here regarding animal handling have been approved by the Institutional Animal Care and Use Committee (IACUC) of the Genome Institute of Singapore and Biological Resource Center of the Agency for Science, Technology and Research, Singapore.

\section{Mouse Infection}

\section{Preparation of glass capillaries}

1. Light an open flame source (Bunsen burner or alcohol burner).

2. Hold a glass capillary with two hands by firmly pinching both ends, then heat the middle of the tube evenly until the glass goes soft. Rotate the capillary gently back and forth along its axis to aid in even heating of the glass.

3. Remove the glass capillary from the heat source and immediately pull hands apart, while maintaining grip on both ends of the tube. The ideal final length of the pulled capillary is $3-5 \mathrm{~cm}$ longer than an unpulled capillary to ensure an appropriate internal diameter for isolating single bladder epithelial cells.

CAUTION: The tubing still remains extremely hot for a period of time, so set the capillary aside on a heat-safe surface for a few minutes to cool before proceeding to the next step.

4. Check to see if the middle of the glass capillary has become narrower (Figure 1A) and that the interior of the tube is still hollow (Figure 1B). For isolation of IBCs, a bore size of $200-400 \mu \mathrm{m}$ is usable.

5. Pick up one end of the pulled capillary with one hand. Hold a pair of forceps with the other hand, and use it to pick up the pulled glass capillary at its narrowest point. Ensure that the forceps are wielded with enough force to grip the capillary firmly without crushing it.

6. Use a rapid twisting motion by the hand holding the forceps to snap the pulled capillary at the narrowest point to create a mouth micropipetting capillary.

NOTE: Using fingers instead of forceps is also acceptable, as long as adequate protection from glass shards is used.

7. Repeat steps 1.1.2-1.1.6 at least $4 \mathrm{x}$ more to produce sufficient spare micropipetting capillaries and provide a range of diameters for IBC isolation. If more than one infection group is anticipated, prepare 5 additional micropipetting capillaries for each additional infection group.

CAUTION: Do not forget to shut off the open flame.

8. Place the pulled capillaries in a $100 \mathrm{~mm}$ Petri dish and expose the dish to UV radiation for $30 \mathrm{~min}$ to sterilize the capillaries.

9. Replace the lid on the Petri dish after UV sterilization and store the petri dish with the capillaries at room temperature.

\section{Preparation of catheters prior to infection}

1. Prepare urinary catheters for infection as described in Hung et al. ${ }^{8}$ and Conover et al. ${ }^{26}$ at least one day before infection.

3. Preparation of fluorescent uropathogenic E. coli culture

1. Grow the selected fluorophore-expressing uropathogenic bacterial strain according to established protocols.

NOTE: The choices of strain and fluorophore largely depend on the microscope and strains available in individual laboratories. In this example, we use a strain derived from UTI89, which is a clinical isolate originally from a patient with recurrent cystitis. This strain, SLC-638, carries a plasmid (pSLC-77) that expresses both vsfGFP-9 and kanamycin resistance ${ }^{18}$. SLC-638 is grown in LB broth at 37 ${ }^{\circ} \mathrm{C}$ supplemented with $50 \mu \mathrm{g} / \mathrm{mL}$ kanamycin.

2. Streak strain SLC-638 on a Luria Bertani (LB)-agar plate supplemented with $50 \mu \mathrm{g} / \mathrm{mL}$ kanamycin. Incubate the plate at $37^{\circ} \mathrm{C}$ overnight.

3. (Optional) View the plate on the dissecting microscope to confirm the expression of fluorescent markers before selecting a colony.

4. Using a bacterial inoculation loop, transfer the selected colony to a $125 \mathrm{~mL}$ conical flask with $10 \mathrm{~mL}$ of LB broth supplemented with 50 $\mu \mathrm{g} / \mathrm{mL}$ kanamycin. Incubate the flask statically at $37^{\circ} \mathrm{C}$ for $24 \mathrm{~h}$.

5. Subculture the bacteria from this flask by taking $10 \mu \mathrm{L}$ of culture from the flask and diluting it in $10 \mathrm{~mL}$ of LB broth supplemented with $50 \mathrm{\mu g} / \mathrm{mL}$ kanamycin in a fresh $125 \mathrm{~mL}$ flask (a 1:1000 dilution). Incubate this second flask statically at $37^{\circ} \mathrm{C}$ for another $24 \mathrm{~h}$.

6. Spin down the bacterial culture for $5 \mathrm{~min}$ at $5,000 \times g$ and $4{ }^{\circ} \mathrm{C}$.

7. Decant the supernatant and resuspend the bacterial pellet in cold $\mathrm{PBS}$ at $\mathrm{OD}_{600}$ of 0.5 .

NOTE: Although it can vary from culture to culture, typically $1 \mathrm{~mL}$ of static culture gives about $4-5 \mathrm{~mL}$ of $O D_{600}=0.5$ bacterial culture. The total volume of bacterial inoculum needed for each strain can be calculated as follows: $50 \mu \mathrm{L}$ is required for each mouse and 50 $\mu \mathrm{L}$ is needed for filling the needle head. An additional $10-20 \%$ (minimum of $50 \mu \mathrm{L}$ ) of the inoculum is recommended to account for dead volume in the syringe.

8. Use the remaining bacterial mixture to determine the infection titer as described in Hung et al. ${ }^{8}$. NOTE: This step may be delayed for a few hours by storing the bacterial mixture at $4{ }^{\circ} \mathrm{C}$.

\section{Murine Model of Urinary Tract Infection}

1. Infect the mice as described by Hung et al. ${ }^{8}$, with one experimental group for each strain of fluorescent E. coli cultured in section 1.3 . NOTE: Also see Conover et al. ${ }^{26}$ for visual assistance.

2. Note the time of bacterial inoculation for the mouse or cage.

3. Repeat infections for the entire experimental group. 
NOTE: The infection catheter may be reused for all mice in the same group.

4. Repeat steps 1.4.1-1.4.3 for each experimental group planned, ensuring that a fresh catheter and new lubricant gel is prepared for every group.

NOTE: For experiments with a large number of animals, it is best to divide the animals into groups of five and staggering the infections such that each group is infected about $30 \mathrm{~min}$ to $1 \mathrm{~h}$ apart. This will provide enough time for the following steps (Sections 2 and 3 ).

\section{Bladder Epithelial Cell Harvesting to Obtain a Cell Suspension}

\section{Harvesting and inverting murine bladders}

1. Prepare three $50 \mathrm{~mL}$ conical tubes filled with $45 \mathrm{~mL}$ of $70 \%$ ethanol for sterilizing surgical equipment.

2. Into two of the tubes prepared in step 2.1.1, place a pair of scissors and a pair of forceps each. Place two pairs of forceps (one preferably narrower and with a rounded tip, for the inversion of the bladder) into the third tube.

NOTE: The tools in the first tube will be used on the external region, the tools in the second will be used in bladder harvesting, and the two pairs of forceps in the last tube will used in bladder inversion.

3. At $6 \mathrm{~h}$ post infection, euthanize the infected mice according the institution's established IACUC protocols.

NOTE: Our IACUC protocol calls for euthanasia via cervical dislocation performed while the mouse is under anesthesia (isoflurane).

4. Lay the animals flat on their backs and use a spray bottle filled with $70 \%$ ethanol to sterilize their abdominal area.

5. Using a pair of forceps and surgical scissors from the first tube (prepared in step 2.1.2), make a small transverse incision on the skin about $1 \mathrm{~cm}$ above the urethral opening. Expand the incision diagonally towards the upper limbs of the mouse, creating a V-shaped cut along the entire anterior of the mouse that exposes the contents of the peritoneum. Ensure that during this process, the scissors do not cut through the intestines of the mouse (Figure 2A,B).

6. Switch to the second set of tools (prepared in step 2.1.2). Using the blades of the scissors or the shafts of the forceps, gently push down on the fat pads near the pelvic region of the mouse. NOTE: This step causes the bladder to protrude outward and ensures visibility for harvesting.

7. Grip the exposed bladder at the apex with a pair of forceps (Figure 2C).

8. Keeping a firm grip on the bladder apex with the forceps, cut and free the bladder from the rest of the animal (cutting the urethra and ureters away) using the surgical scissors. Do not release the forceps holding the bladder yet.

9. Switching from the scissors to the narrower rounded forceps from the third conical tube (from step 2.1.2), insert the tip of one shaft of the rounded forceps into the opening of the bladder where it was just cut in the previous step (Figure 2D). With the tip of the rounded forceps safely inserted into the opening of the bladder, release the pair of forceps gripping the apex of the bladder and return it to the second conical tube.

10. Using the second pair of forceps from the third tube, gently turn the bladder "inside out", first pulling the outside end of the mouth of the bladder away from the rounded forceps (Figure 2D, arrow 1) and guiding it around and over the other tip of the rounded forceps (Figure 2D, arrow 2).

NOTE: The action can be likened to removing a sock from one foot and pulling it over the other.

1. During the inversion process, maintain the first rounded pair of forceps almost completely closed. This provides enough freedom of movement to pull the bladder off from the first shaft of the rounded forceps, but also brings the second shaft of the rounded forceps closer to the first and allows for the bladder to be transferred easily. The final result of this step is that the bladder should end up being inverted and on the tip of the second shaft of the first pair of forceps (Figure 2E).

2. Using the second pair of forceps, gently coax the inverted bladder off the tip of the forceps into $1 \mathrm{~mL}$ of cold PBS. NOTE: (Optional) This is the opportune time to take images of the entire inverted, infected bladder to observe the general frequency and distribution of IBCs, if any.

11. Repeat steps 2.1.1-2.1.10 for each bladder in the experimental group (to a maximum of five animals).

\section{Bladder epithelial cell scraping}

1. Using two clean pairs of forceps, gently scrape the outside of the inverted bladder (which is the internal epithelial cell layer). The surrounding PBS should appear cloudier as scraping proceeds and epithelial cells are released into the PBS solution.

2. (Optional) Visually confirm that the bladder scraping has released cells into solution using a dissecting microscope. Figure 3A,B). The PBS should appear cloudy to the naked eye, and the scraped individual bladder epithelial cells can be seen at 10x magnification.

3. Repeat steps 2.2.1-2.2.2 for each harvested bladder from step 2.1 .

\section{Intracellular Bacterial Community (IBC) isolation: Mouth Pipetting of IBCs}

NOTE: All methods described in this section have undergone an institutional risk assessment. Mouth pipetting carries the inherent risk of ingestion of the solution that is being transferred. This risk is largely mitigated by the nanoliter volumes that this protocol uses, and we recommend that all users of the protocol pay heed to the precautionary and practice notes listed here and in the discussion.

1. After the cells have been scraped into the PBS, set up the mouth micropipetting apparatus (Figure 3C).

1. Insert the thicker end of the pulled glass capillary (the unpulled end) into the rubber plug (white end) of the aspirator tube.

2. Insert the narrow end of a $1 \mathrm{~mL}$ pipette tip into the other open (red) end of the aspirator tube, ensuring that there is a tight fit.

3. Insert the narrow end of a $2 \mathrm{~mL}$ aspirating pipette into the open, wider end of the $1 \mathrm{~mL}$ pipette tip, again ensuring that there is a tight fit. NOTE: The resulting setup allows the researcher to mouth pipette from the wider, open end of the aspirating pipette to create a gentle suction force from the narrow end of the capillary tube at the other end of the apparatus.

4. Test the final mouth micropipetting apparatus using a $100 \mathrm{~mm}$ Petri dish containing fresh deionized water. A slight suction action on the open end of the aspirating pipette (similar to sipping on a drink through a straw) should increase the level of liquid in the capillary, but 
not cause the deionized water to overflow into the aspirator tube. Use one hand to control the capillary tube, while using the other hand to adjust the position of the Petri dish.

NOTE: The strength of suction required for mouth-pipetting a single IBC will vary among researchers. However, it is recommended that each researcher attempting this technique start with a weak suction and slowly increase it if no liquid is flowing up the capillary. There is no need for a force greater than sucking on a straw for drinking. If the capillary does not appear to be picking up liquid during the test in step 3.1.4, it is possible that either the capillary or the aspirating tube is occluded and needs to be replaced. It is further recommended that all new researchers first practice controlling suction in the mouth pipetting apparatus using sterilized water. Additionally, note that the control of the volume taken up by the mouth pipetting apparatus is through the use of the researcher's tongue. The tongue can finely adjust the strength of suction applied, as well as act as an emergency stop.

5. After achieving successful uptake of liquid, test the ability to expel it from the capillary by gently blowing into the open end of the aspirating pipette. Ensure that no bubbles are created in the process of expelling the liquid to prevent contamination of the IBCs during step 3.5 .

NOTE: As with suction, the strength of positive pressure applied by the researcher to expel the IBC into the centrifuge tube will vary among researchers. It is recommended for researchers new to this protocol to practice step $3.1 \mathrm{a}$ few days before the actual infection. One suggestion for practicing mouth micropipetting is to practice transferring small volumes of sterilized water mixed with a few drops of food dye (for visibility) using the mouth micropipette apparatus.

2. Place the scraped cell suspension under the dissecting microscope and identify the IBCs as large fluorescent aggregates (Figure $\mathbf{3 A}, \mathbf{B})$. The ideal range of magnification is 20-40x. Dip the fine end of the glass capillary into a fresh tube of PBS for $1 \mathrm{~s}$ to reduce the uptake of unwanted volume through capillary action.

3. Looking through the microscope, identify the IBC of interest and slowly bring the open end of the capillary tube toward the IBC. Use the fine end of the glass capillary to sweep away extra cells near each IBC to prevent the aspiration of two or more cells, or to break apart larger aggregates of cells.

4. While looking through the microscope, apply a very small suction force on the far end (aspirating pipette) of the mouth micropipetting apparatus to guide the IBC into the glass capillary.

5. After picking up the $\mathrm{IBC}$, move the capillary to an empty $1.5 \mathrm{~mL}$ centrifuge tube and apply a slight positive pressure to expel the droplet and the IBC into the centrifuge tube (Figure 3D).

6. Repeat steps 3.3-3.6 on as many IBCs are needed from the current bladder, before proceeding to the next bladder. Change aspirating pipettes and capillaries frequently to prevent build-up of saliva.

CAUTION: When working with infectious (or clinical) strains of bacteria, constantly monitor the level of solution in the capillary. Do not let the level of liquid being pipetted overflow from the edge of the capillary into the aspirator tube. If this occurs, immediately switch to a different aspirator tube, and discard the previous set up.

7. Repeat steps 3.2-3.6 on all harvested bladders, or until sufficient number of biological samples have been collected for the experimental group.

8. (Optional) Repeat steps 3.6 and 3.7 until all experimental groups (or mice) have been euthanized and sufficient biological samples have been harvested from each group.

\section{Representative Results}

Apart from confirmation (Figure 3D) of the presence of a single isolated IBC in the collection tube via the dissecting microscope, the purity of the isolated IBC can also be confirmed by confocal microscopy. As shown in Figure 4A, the isolated cells should stain for both $E$. coli and uroplakin, and are the expected size for IBCs $(50-120 \mu \mathrm{m})^{17}$. Furthermore, E. coli staining is not present in the surrounding liquid. Based on our data, more than $90 \%$ of cells isolated with this technique are IBCs ${ }^{18}$. After isolation, the presence and viability of the bacterial cells in the individual IBC can be confirmed through colony forming unit (CFU) enumeration (Figure 4B) or quantitative polymerase chain reaction (qPCR) for genomic equivalents (Figure 4C). Figure 4C also demonstrates that uninfected epithelial cells isolated with the same protocol do not have quantifiable amounts of bacteria. Based on these data, we estimate that the range of CFUs in a single IBC is $10^{2}-10^{3}$ in the murine model of urinary tract infection. One of the main goals of single IBC isolation is to perform downstream analyses such as RNA sequencing. To verify that our isolation method is able to obtain RNA from bacteria in IBCs for analysis, we performed quantitative reverse transcription polymerase chain reaction (qRTPCR) quantification of three genes (16S, cyoB, and frdA) for a range of individually isolated and pooled IBCs (Figure 4D). All the data shown in Figure 4 has been adapted with permission from Duraiswamy et al. ${ }^{18}$. An overview schematic of our IBC isolation protocol can be seen in Figure 5 , which is reproduced from Duraiswamy et al. ${ }^{18}$. 


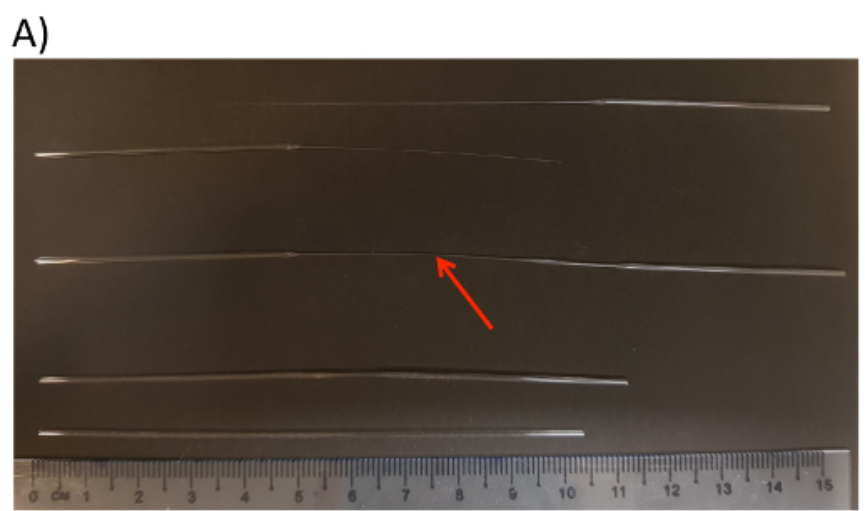

B)

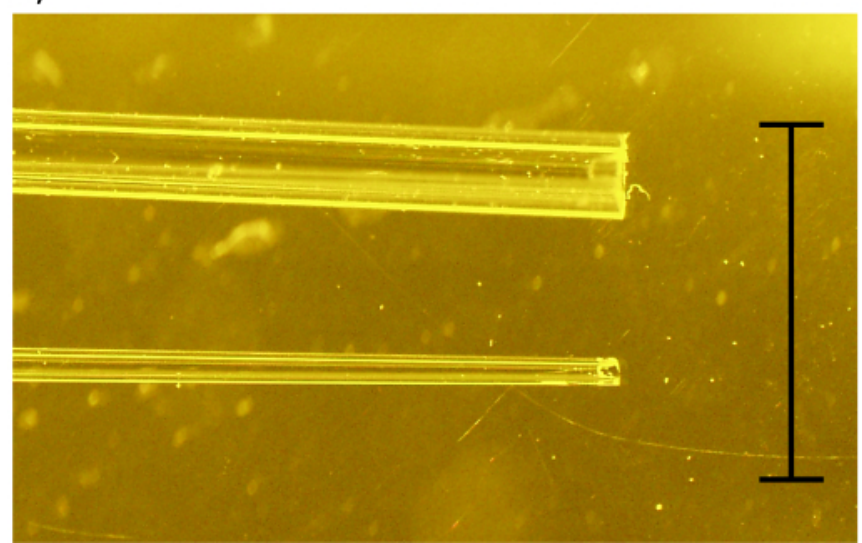

Figure 1: Hand-pulled capillaries retain narrow openings. (A) Samples of hand-pulled capillary tubes are displayed on a black background for contrast. From bottom to top, an unpulled capillary, a capillary that was not pulled to a sufficient extent, a capillary that can be used for single bladder epithelial cell harvesting, and a capillary that was pulled too thin (and thus separated into two pieces) are shown. A $15 \mathrm{~cm}$ ruler is placed at the bottom of the image for scale. The estimated point for snapping off the usable capillary is indicated on the figure by the red arrow. (B) Image taken with a dissecting microscope confirming the hollow internal diameter of a pulled capillary (bottom). An unpulled capillary is positioned above to demonstrate the relative size difference of the two capillaries. Scale bar $=4.0 \mathrm{~mm}$. Please click here to view a larger version of this figure. 
A)

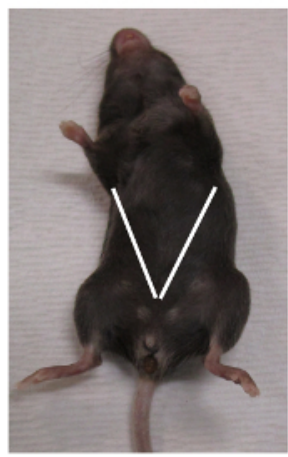

B)

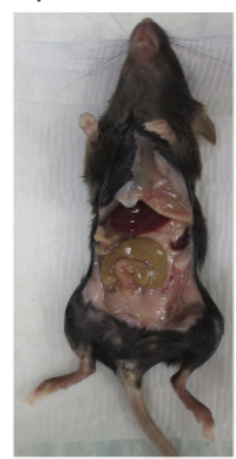

C)

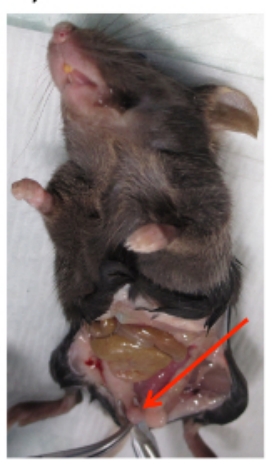

D)

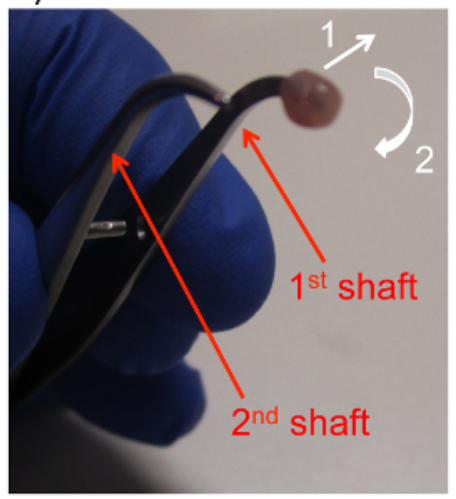

E)

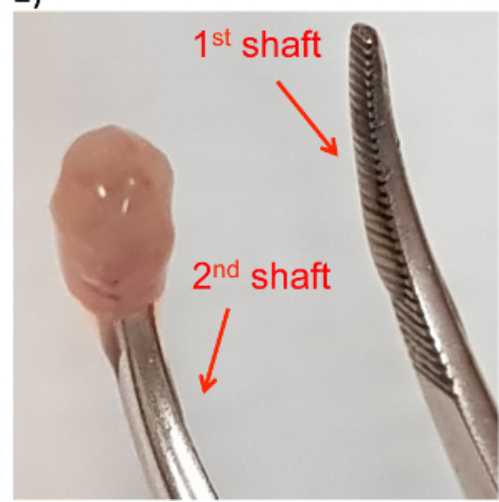

Figure 2: Dissection of mouse to harvest bladder epithelial cells. (A) An image of a mouse with white lines added to indicate the estimated location and angle of incisions to expose the murine peritoneal cavity and bladder. (B) An image of the exposed mouse peritoneal cavity postincision. (C) An image of the exposed bladder (red arrow) protruding from between the fat pads. (D) An image of the murine bladder with the tip of the forceps inserted into the lumen, with arrows to indicate the direction of motion needed to invert the bladder. The bladder is first pulled slightly outwards, then around and off the first shaft of the forceps. The directions of motion for both actions are as indicated by white arrows numbered 1 and 2. (E) An image showing the final position of the inverted bladder inserted onto the second shaft of the forceps. The shafts of the forceps are labeled in both panels $D$ and $E$ with red arrows and text. Please click here to view a larger version of this figure. 
A)

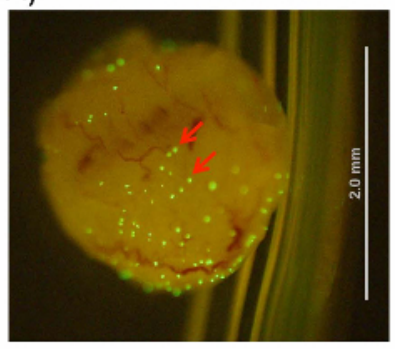

C)

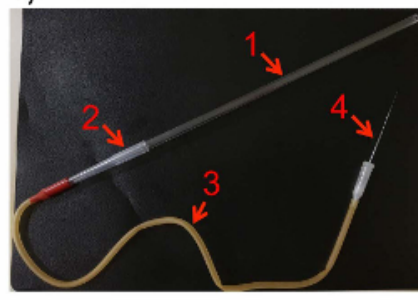

Parts of a mouth micropipette:

1) $2 \mathrm{~mL}$ aspirating

pipette

2) $1 \mathrm{~mL}$ pipette tip

3) Aspirator tube

4) Pulled capillary tube

D)

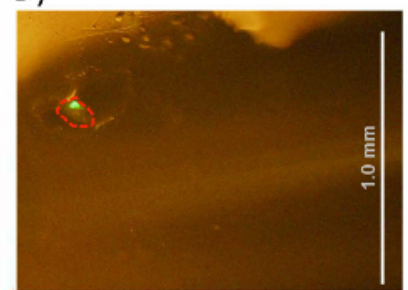

Figure 3: IBC harvesting from bladder cells. (A) An infected and inverted bladder in cold PBS solution before cell scraping. (B) An image showing scraped bladder cells as seen under a microscope. IBCs can be identified as large green fluorescent aggregates in both images (see red arrows). (C) An image of the completed mouth micropipetting apparatus. The aspirating pipette, pipette tip, aspirator tube, and pulled capillary tube are identified with numbered arrows as indicated on the right. (D) An image of a single isolated IBC within a $1.5 \mathrm{~mL}$ collection tube (outlined in red). Scale bars (as indicated) are represented by white lines in panels A, B, and D. Please click here to view a larger version of this figure. 
A)

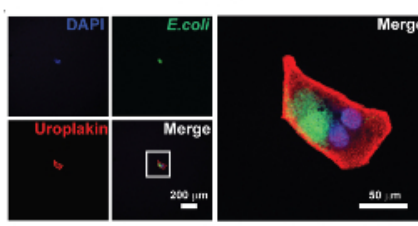

IBC 2

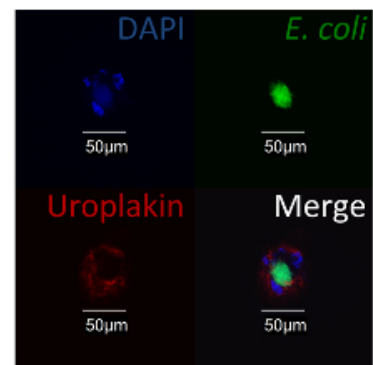

C)

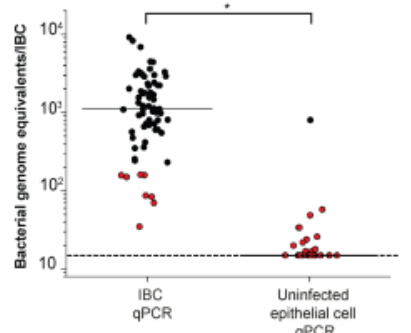

D)
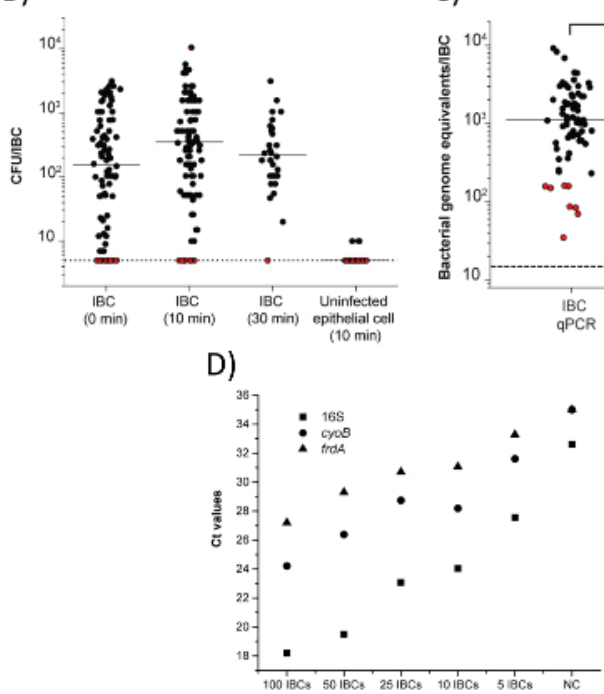

Figure 4: Harvested IBCs are pure and can be used for downstream analysis. This figure has been modified with permission from Duraiswamy et $\mathrm{al}^{18}$. (A) Images of two isolated GFP-positive cells that were stained with anti-uroplakin and anti-E.coli antibodies. The first cell (IBC 1) has images of individual channels (at low-magnification) on the left, and a high-magnification merged image is on the right. The second cell (IBC 2) is shown in high magnification in merged and individual channels. Scale bars are as indicated. DNA is stained with 4',6-diamidino-2-phenylindole (DAPI) and represented in the blue channel. Anti- $E$. coli is stained with a secondary antibody conjugated to fluorescein isothiocyanate (FITC) and represented in the green channel. Anti-uroplakin is stained with a secondary antibody conjugated to tetramethylrhodamine isothiocyanate (TRITC) and represented in the red channel. (B) Bacterial CFUs from isolated IBCs. IBCs were processed immediately, or incubated in $0.1 \%$ Triton-X for 10 or $30 \mathrm{~min}$. Pooled CFU counts of individual IBCs isolated from $\mathrm{n}=3$ separate experiments are shown. Limit of detection $=0.7 \log _{10}$ CFUs/IBC. Red dots plotted at the limit of detection indicate samples for which no colonies were recovered. All IBC-containing samples are not significantly different $(p>0.05$, Mann-Whitney test); the uninfected epithelial cells are significantly different from the IBC (10 $\mathrm{min})$ data $(p<0.001$, Mann-Whitney test). (C) qPCR quantification of bacteria on individual IBCs and uninfected epithelial cells after a 10 min incubation in $0.1 \%$ Triton- $X\left({ }^{*}, p<0.0001\right.$, Mann-Whitney test, $\left.n=4\right)$. Limit of detection $=1.18$ log $_{10}$ bacterial genome equivalents $/$ IBC. Red dots indicate samples for which no colonies were recovered on titering in panel B. (D) Quantification of the 16S rRNA, cyoB, and frdA genes for varying numbers of individually isolated and pooled IBCs $(n=1$ experiment; each point indicates the mean of 3 technical replicates). $\mathrm{NC}=$ no DNA negative control. Please click here to view a larger version of this figure. 


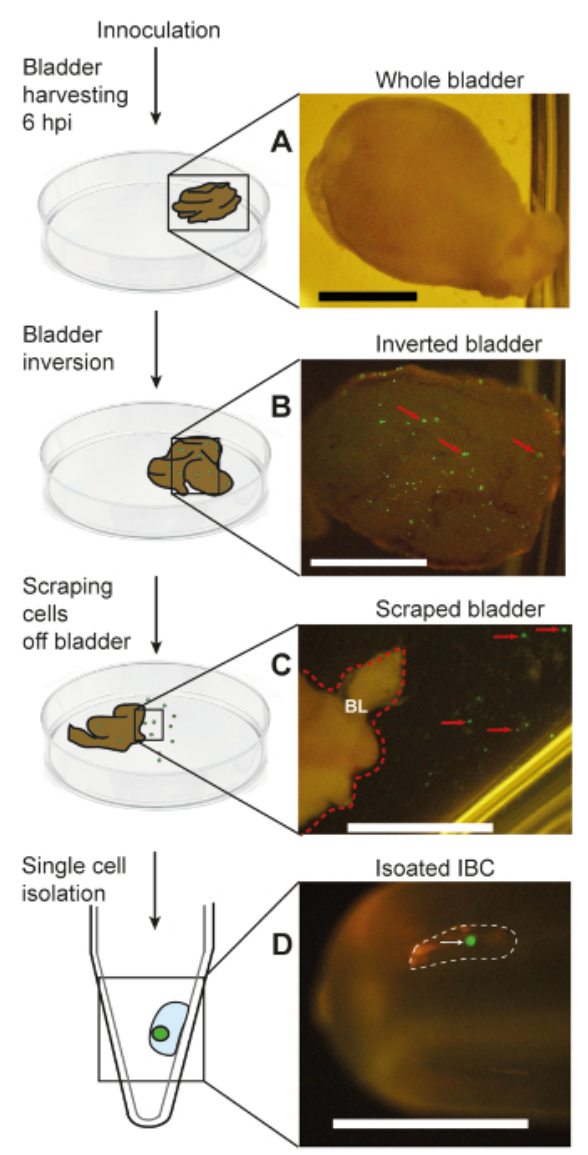

Figure 5: A schematic and its associated photographs representing the isolation of IBCs via mouth micropipetting from infected mice bladders. This figure is reproduced from Duraiswamy et al. ${ }^{18}$. (A) A harvested whole bladder; (B) an inverted whole bladder exposing the GFP expressing IBCs; (C) a close-up of the edge of a scraped bladder showing individual IBCs in suspension in the adjacent buffer; (D) a single isolated IBC pipetted into a tube. Red arrows in panel B indicate examples of GFP-positive IBCs on the luminal surface of the bladder. The red dotted line in panel $C$ indicates the right border of the inverted bladder (indicated as "BL"); red arrows in panel C indicate apparent individual GFP-positive epithelial cells that have been scraped off the bladder surface. White dotted line in panel D indicates a micropipetted sub-microliter droplet containing an isolated IBC, which is indicated by a white arrow. Scale bars $=2 \mathrm{~mm}$. Please click here to view a larger version of this figure.

\section{Discussion}

The protocol we have described allows for the isolation of single IBCs from a murine model of UTI. This protocol isolates IBCs containing viable intracellular bacteria, which can be verified by culturing for CFU. The protocol results in intracellular bacteria from IBCs with little contamination by extracellular bacteria, allowing for further characterization of both bacteria and host cell from an IBC (Figure 4C). We also show that the bacteria from a single IBC can be used in downstream applications such as qPCR (Figure 4C), suggesting that our technique can be used to process IBCs for other in vitro analyses. By pooling the bacteria harvested from as few as 5 IBCs, we further demonstrate our ability to perform qRT-PCR analysis on three bacterial genes, suggesting that good quality RNA can be harvested from the bacteria in our isolated IBCs (Figure 4D). Combined, the data we have shown indicate that performing genome-wide RNA analysis (such as RNA sequencing) on single IBCs may be possible using this isolation technique.

In this protocol, we have focused on the $6 \mathrm{~h}$ time point because that is when IBC numbers peak in the bladders of black 6 mice infected by UTI89 27 . Furthermore, we have also used a static bacterial culture system to enhance the level of type 1 pilus expression in UTI89. The expression of type 1 pilus is critical for $E$. coli to attach to and infect bladder epithelial cells ${ }^{28}$. However, this expression is tightly regulated ${ }^{29}$ and environmental cues are known to alter it ${ }^{30}$. In order to maintain a consistent infection phenotype and sufficient numbers of IBCs, we recommend using a $2 \times 24 \mathrm{~h}$ static bacterial culture (slightly modified from Hung et al. ${ }^{8}$ ) and the $6 \mathrm{~h}$ infection time point when working with previously tested E. coli strains such as NU14 and UTI89 ${ }^{28,29}$. However, it is possible that these variables will need to be adjusted in other UTI strains or in other mice strains to obtain the ideal number of IBCs from each infection.

While the protocol from Hung et al. ${ }^{8}$ uses only female mice, other established protocols for establishing urinary tract infection in male mice have been reported ${ }^{31}$. In this model, cystitis in male mice also followed the IBC pathway. As the bladders of male and female mice are similar in size, we anticipate that our IBC isolation protocol can be used on infected male mice as well.

The relatively simple technology utilized in this protocol also ensures that it can be deployed in most laboratories. One of the key steps involved in this protocol is the pulling of glass capillaries to create microcapillaries for selecting the cell type of interest. This step allows for flexibility 
in the diameters of microcapillaries created, and thus the method can be extended to multiple different target cell types. However, due to the inherent variation in creating these capillaries, care must be taken to ensure that the final diameter is in a usable range. If the capillaries are too narrow, they fail to pick up the cell of interest, but if they are made too wide, multiple cells could be selected in a single attempt. Furthermore, the use of an open flame during the process of capillary pulling carries an inherent risk of burns and fire, so the researcher attempting to create microcapillaries should take care to prevent such events from occurring. To reduce the variability as well as the open fire risk involved in making these capillaries, the researcher could make use of a traditional micropipette pulling machine, such as those used for electrophysiological experiments (e.g., PC-100, Narishige group). As these machines make use of either gravity or robotic platforms to pull the capillaries, they can be tailored to meet the needs of the infection model. However, the wide range of micropipette pulling machines available will mean that the individual researcher will need to go through some trial and error to determine the appropriate final capillary diameter for use with this protocol.

The presented protocol makes use of bacteria expressing a fluorescent tag to visually identify the IBC. Thus, this technique is limited by the researchers' ability to genetically modify the infectious organism. Specifically for UPEC, IBC-forming strains such as CFT073 and NU14 have been successfully transformed with GFP-expressing plasmids ${ }^{32,33,34}$; these should therefore be usable in the same protocol. Based on the area of the mouse bladder $\left(70 \mathrm{~mm}^{2}\right)^{35}$, the length of individual epithelial cells $(50-120 \mu \mathrm{m})^{17}$, and the frequency of IBCs in a single bladder ${ }^{16}$, a conservative estimate for the incidence of IBCs is about 1 in 1,000 cells (or $0.1 \%$ ). This estimate showcases the utility of our cell isolation protocol to target rare events. The precision of cell selection through our protocol and the wide range of capillary diameters that can be pulled suggest that this protocol can be used to isolate intracellular bacteria from other in vivo and in vitro models of infection. Indeed, we have successfully used this technique to isolate infected cultured bladder epithelial cells (data not shown).

One of the more technically challenging steps in the protocol is inverting the bladder to expose the epithelial cells for scraping. We have found that it is also possible to make an incision on the bladder to splay it out for scraping. However due care should be taken to reduce the damage to the epithelial cells of the bladder during the process of cutting it open; ideally a single cut should be utilized to splay the bladder open. Additionally, the cut should be made in cold PBS, to prevent accidental loss of cells or bladder tissue during the process.

Mouth pipetting of the IBCs in this protocol provides greater control over the cell selection process, as well as limiting the final volume of solution transferred together with the cell. The fine control and large separation of solution from the researchers' mouth also maximizes the safety of the researcher, as the volumes transferred are within the nanoliter to microliter range. In contrast, our experience with the modern micropipette is that it tends to transfer more surrounding liquid and cells with the IBC, potentially leading to contamination with extracellular luminal bacteria. Our finding that mouth pipetting provides a higher performance over other single cell isolation methods has also been reported by other labs ${ }^{22,23,24}$. Aside from single cell isolation, mouth pipetting has even been used in single-cell electroporation for neurons ${ }^{25}$, which further demonstrates the utility and the minute control that a trained researcher can attain with the technique. However, safety is of primary importance, and we suggest potential additional measures that can be taken depending on the pathogens being used: (i) the extension of the air buffer between the researcher and the biological material, for example by using an aspirating pipette with a larger volume (e.g., $5 \mathrm{~mL}$ ), or (ii) adding a physical filter such as cotton wool into the aspirating pipette to act as an additional barrier.

In cases where a risk assessment leads to the conclusion that mouth pipetting is still too risky, commercially available robotic setups (such as those used for nanoinjections) can be combined with the other sections of our technique to provide a safer method for isolating infected cells from mixed populations. It should be noted that our experience with using a robotic micromanipulator has demonstrated a decreased rate of IBC isolation compared to mouth pipetting, as the large intra-experimental variation in bladder epithelial cell size makes it challenging for the user of a robotic arm to determine the force required to pick up single IBCs. Nevertheless, it remains a viable, though costlier, option for those working with highly infectious agents of disease.

\section{Disclosures}

The authors have nothing to disclose.

\section{Acknowledgments}

This research was supported by the National Research Foundation, Prime Minister's Office, Singapore, under its NRF Research Fellowship Scheme (NRF award no. NRF-RF2010-10); the Singapore Ministry of Health's National Medical Research Council (NMRC/CIRG/1358/2013); and the Genome Institute of Singapore (GIS)/Agency for Science, Technology, and Research (A*STAR).

\section{References}

1. Barber, A. E., Norton, P. J., Spivak, A. M., Mulvey, M. A. Urinary Tract Infections: Current and Emerging Management Strategies. Clinical Infectious Diseases. 57 (5), 719-724 (2013).

2. Flores-Mireles, A. L., Walker, J. N., Caparon, M., Hultgren, S. J. Urinary tract infections: epidemiology, mechanisms of infection and treatment options. Nature Reviews Microbiology. 13, 269 -284 (2015).

3. Foxman, B. Recurring urinary tract infection: incidence and risk factors. American Journal of Public Health. 80, 331-333 (1990).

4. Foxman, B., Barlow, R., D'Arcy, H., Gillespie, B., Sobel, J. D. Urinary tract infection: self-reported incidence and associated costs. Annals of Epidemiology. 10 (8), 509-15 (2000).

5. Zowawi, H. M. et al. The emerging threat of multidrug-resistant Gram-negative bacteria in urology. Nature Reviews Urology. 12, 570-584 (2015).

6. Silverman, J. A., Schreiber, H. L. IV, Hooton, T. M., Hultgren S. J. From physiology to pharmacy: developments in the pathogenesis and treatment of recurrent urinary tract infections. Current Urology Reports. 14, 448 - 456 (2013).

7. Sivick, K. E., Mobley, H. L. T. Waging war against uropathogenic Escherichia coli: winning back the urinary tract. Infection and Immunity. 78, $568-585(2010)$.

8. Hung, C.-S., Dodson, K. W., Hultgren, S. J. A murine model of urinary tract infection. Nature Protocols. 4, 1230-1243 (2009). 
9. Cusumano, C. K. et al. Treatment and prevention of urinary tract infection with orally active FimH inhibitors. Science Translational Medicine. (2011).

10. Alteri, C. J., Hagan, E. C., Sivick, K. E., Smith, S. N., Mobley, H. L. T. Mucosal Immunization with Iron Receptor Antigens Protects against Urinary Tract Infection. PLoS Pathogens. (2009).

11. Mysorekar, I. U., Hultgren, S. J. Mechanisms of uropathogenic Escherichia coli persistence and eradication from the urinary tract. Proceedings of the National Academy of Sciences of the United States of America. (2006).

12. Hunstad, D. A., Justice, S. S. Intracellular lifestyles and immune evasion strategies of uropathogenic Escherichia coli. Annual Review of Microbiology. 64, 203-221 (2010).

13. Rosen, D. A., Hooton, T. M., Stamm, W. E., Humphrey, P. A., Hultgren, S. J. Detection of intracellular bacterial communities in human urinary tract infection. PLoS Medicine. (2007).

14. Robino, L. et al. Detection of intracellular bacterial communities in a child with Escherichia coli recurrent urinary tract infections. Pathogens and Disease. 68 (3), 78-81. (2013).

15. Robino, L. et al. Intracellular bacteria in the pathogenesis of Escherichia coli urinary tract infection in children. Clinical Infectious Diseases. $\mathbf{5 9}$ (11), e158-64. (2014).

16. Schwartz, D. J., Chen, S. L., Hultgren, S. J., Seed, P. C. Population dynamics and niche distribution of uropathogenic Escherichia coli during acute and chronic urinary tract infection. Infection and Immunity. 79, 4250 - 4259 (2011).

17. Keshtkar, A., Keshtkar, A., Lawford, P. Cellular morphological parameters of the human urinary bladder (malignant and normal). International Journal of Experimental Pathology. 88, 185-190 (2007).

18. Duraiswamy, S., Chee, J. L. Y., Chen, S., Yang, E., Lees, K., Chen, S. L. Purification of Intracellular Bacterial Communities during Experimental Urinary Tract Infection Reveals an Abundant and Viable Bacterial Reservoir. Infection and Immunity. (2018).

19. Kurimoto, K., Yabuta, Y., Ohinata, Y., Saitou, M. Global single-cell cDNA amplification to provide a template for representative high-density oligonucleotide microarray analysis. Nature Protocols. 2, 739-752 (2007).

20. Tang, F. et al. Deterministic and stochastic allele specific gene expression in single mouse blastomeres. PLoS One. (2011).

21. Wells, J. M., Melton, D. A. Early mouse endoderm is patterned by soluble factors from adjacent germ layers. Development. 127, 1563-1572 (2000).

22. Guo, H. et al. Profiling DNA methylome landscapes of mammalian cells with single-cell reduced-representation bisulfite sequencing. Nature Protocols. 10 (5),645-59 (2015).

23. Zhao, R. et al. The establishment of clonally derived chicken embryonic fibroblast cell line (CSC) with high transfection efficiency and ability as a feeder cell. Journal of Cellular Biochemistry. (2018).

24. Tang, F. et al. RNA-Seq analysis to capture the transcriptome landscape of a single cell. Nature Protocols. 5 (3), $516-35$ (2010).

25. Wiegert, J. S., Gee, C. E, Oertner, T. G. Single-Cell Electroporation of Neurons. Cold Spring Harbor Protocols. (2017).

26. Conover, M. S., Flores-Mireles, A. L., Hibbing, M. E., Dodson K, Hultgren, S. J. Establishment and Characterization of UTI and CAUTI in a Mouse Model. Journal of Visualized Experiments. (100), e52892. (2015).

27. Justice, S. S. et al. Differentiation and developmental pathways of uropathogenic Escherichia coli in urinary tract pathogenesis. Proceedings of the National Academy of Sciences of the United States of America. 101 (5), 1333-8 (2004).

28. Mulvey, M. A et al. Induction and Evasion of Host Defenses by Type 1-Piliated Uropathogenic Escherichia coli. Science. 282 (5393), 1494-7 (1998).

29. Zhang, H., Susanto, T. T., Wan, Y., Chen, S. L. Comprehensive mutagenesis of the fimS promoter regulatory switch reveals novel regulation of type 1 pili in uropathogenic Escherichia coli. Proceedings of the National Academy of Sciences of the United States of America. 113 (15), 4182-7. (2016).

30. Gally, D. L., Bogan, J. A., Eisenstein, B. I., Blomfield, I. C. Environmental regulation of the fim switch controlling type 1 fimbrial phase variation in Escherichia coli K-12: effects of temperature and media. Journal of Bacteriology. 175 (19), 6186-93 (1993).

31. Olson, P. D., Hruska, K. A., Hunstad, D. A. Androgens Enhance Male Urinary Tract Infection Severity in a New Model. Journal of the American Society of Nephrology. 27 (6), 1625-34. (2016).

32. Garofalo, C. K et al. Escherichia coli from Urine of Female Patients with Urinary Tract Infections Is Competent for Intracellular Bacterial Community Formation. Infection and Immunity. 75 (1),52-60 (2007).

33. Berry, R. E, Klumpp, D. J., Schaeffer, A. J. Urothelial cultures support intracellular bacterial community formation by uropathogenic Escherichia coli. Infection and Immunity. 77 (7),2762-72 (2009).

34. Holden, N., Totsika, M., Dixon, L., Catherwood, K., Gally, D. L. Regulation of P-fimbrial phase variation frequencies in Escherichia coli CFT073. Infection and Immunity. 75 (7),3325-34 (2007).

35. Jost, S. P. Postnatal growth of the mouse bladder. Journal of Anatomy. 143, 39-43 (1985). 